\title{
STUDY ON CONSTRUCTION OF 3D BUILDING BASED ON UAV IMAGES
}

\author{
Xie Feifei ${ }^{\mathrm{a}, \mathrm{b},}$, , Lin Zongjian ${ }^{\mathrm{b}}$, Gui Dezhu ${ }^{\mathrm{c}}$, Lin Hua $^{\mathrm{d}}$ \\ ${ }^{a}$ School of Remote Sensing and Information Engineering, Wuhan University, Wuhan, China-xiefeifei_007@163.com \\ ${ }^{\mathrm{b}}$ Chinese Academy of Surveying and Mapping, Beijing, China-lincasm@casm.ac.cn \\ ${ }^{\mathrm{c}}$ Development Research Center for Surveying \& Mapping, State Bureau of Surveying \& Mapping, Beijing, China. - \\ guidezhu0930@163.com \\ ${ }^{\mathrm{d}}$ Guizhou Guihang Unmanned Aerial Vehicles Limited Liability Company, 87 West City Road, AnShun, China- \\ gzwrj@vip.163.com
}

\section{COMMISSION I, ICWG I/V}

KEY WORDS: City, Image, Camera, Mosaic, Texture, System, Test

\begin{abstract}
:
Based on the characteristics of Unmanned Aerial Vehicle (UAV) system for low altitude aerial photogrammetry and the need of three dimensional (3D)city modeling, a method of fast 3D building modeling using the images from UAV carrying four-combined camera is studied. Firstly, by contrasting and analyzing the mosaic structures of the existing four-combined cameras, a new type of four-combined camera with special design of overlap images is designed, which improves the self-calibration function to achieve the high precision imaging by automatically eliminating the error of machinery deformation and the time lag with every exposure, and further reduce the weight of the imaging system. Secondly, several-angle images including vertical images and oblique images gotten by the UAV system are used for the detail measure of building surfaces and the texture extraction. Finally, two tests that are aerial photography with large scale mapping of 1:1000 and 3D building construction in Shandong University of Science and Technology and aerial photography with large scale mapping of 1:500 and 3D building construction in Henan University of Urban Construction, provide authentication model for construction of 3D building based on combined wide-angle camera images from UAV system. It is demonstrated that the UAV system for low altitude aerial photogrammetry can be used in the construction of 3D building production, and the technology solution in this paper offers a new, fast and technical plan for the $3 \mathrm{D}$ expression of the city landscape, fine modeling and visualization.
\end{abstract}

\section{INTRODUCTION}

Three-dimensional simulation and virtual reality are two forms of "digital city" and in the building forms both of them are required establishing $3 \mathrm{D}$ model. Building modeling method is mainly divided in two ways, which are high precision and fast modeling for building group and fine modeling for one building to update the building area. Nowadays, the advanced technology of remote sensing and photographic measurement technology is an important means of 3D building modeling. Because of the limitation of flying high, photography posture and timeliness, fixed orbit satellite remote sensing and manned aerial photography in middle and high altitude can only get the information of the height and top textures of some buildings, which are hard to meet the requirement of $3 \mathrm{D}$ data rapid access and update for city building (Wu Jun, 2003; Li Chengming, 2008).

In recent years, UAV system for low altitude aerial photogrammetry with its unique advantage gets more and more attention from the photogrammetry industry and expands the practical application scope. Now, using multiple cameras combined to form an equivalent large plane array camera is the world advanced technology achievements(National Remote Sensing Center,2009).Combined wide-angle camera images from UAV system increases the base-height ratio to improve the quantity of height measurement with the wide angle of view from the longitudinal direction, and with the wide angle of view from the lateral direction it increases the surface width covered in the images to raise the efficiency of the flight and reduce the quantity of the control points laid in the outdoors. With certain route planning, every part of survering area can be photography with more than four images by the UAV system with combined wide-angle camera, including vertical images and oblique images, which can effectively avoid the influence of the dodging from each building to meet the requirements of 3D data for city building.

Based on the characteristics of UAV system for low altitude aerial photogrammetry, a method of fast $3 \mathrm{D}$ building modeling using the images from UAV carrying four-combined camera is researched. Firstly, the basic idea and technical process are described as below, and then the key technologies are discussed. Finally, two tests are done which show the proposed method feasible.

\section{THE BASIC IDEA AND TEHCHNICAL PROCESS}

Firstly, the structure of special light four-combined camera using the self-calibration technology is designed, which can solve the contradiction between the mechanical stability and volume reduction, the position stabilization and the weight lighting . The four-combined image which is projected into a precise equivalent center from four single camera images by the self-calibration technology, are used for the high precision aerial triangulation to get their accurate exterior orientation elements. Then, according to the relationship between the single camera image and four-combined image, the accurate exterior orientation elements of the single camera image can be

\footnotetext{
* Corresponding author.
} 
calculated. Finally, the measurement of building details with multidimensional aspects is done, including texture image position, optimized selection, high accuracy registration and local distortion. The technology process of UAV loading fourcombined camera for 3D building construction is show in Fig.1.

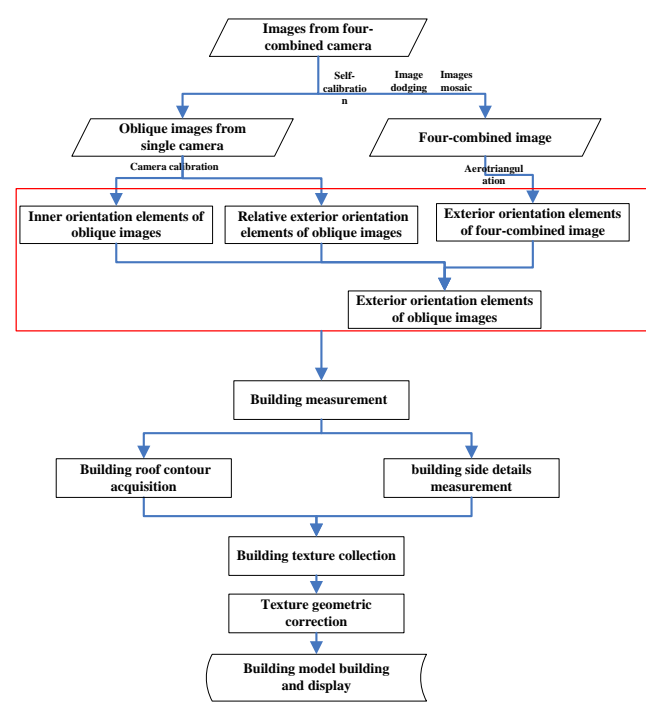

Figure 1. The technology roadmap

\section{RESEARCH AND ANALYSIS THE KEY TECHNOLOGY}

\subsection{The design of four-combined wide-angle camera structure}

There are some mature products of four-combined wide-angle camera at home and abroad, i.e., the international advanced product DMC and UCD, whose mechanical structures are shown in Fig.2a and Fig.2b. The narrow field angle in these structures leads to aerial photography measurement with low elevation precision, according to report, which accuracy can only meet the requirements with the scale mapping of 1:2000. The Chinese product SWDC, whose mechanical structure is shown in Fig.3c, has wide view angle. In order to avoid and eliminate the errors caused by the mechanical deformation and the synchronization exposure time difference, it can only rely on the strong stability of the mechanical structure and the high accuracy of the exposure control circuit (Peng Xiaodong, 2009), so the camera system must be designed with large volume and more than $100 \mathrm{~kg}$ weight, which is not suitable for the small UAV.

The structure of the four-combined wide-angle camera designed by the Chinese Academy of Surveying and mapping is shown in Fig.3. Through the comparison of some digital camera performances, the camera of Cannon EOS 5D Mark II is selected for the four-combined camera. The biggest view angle of the four-combined camera can reach to $130^{\circ}$. The selfcalibration technology is used in the overlap areas of four single camera images to eliminate the errors caused by the mechanical deformation and the synchronization exposure time difference, which can complete the mosaic to a four-combined image (Lin Zongjian, 2011). At the same time, the platform stabilizing device can be given up to further reduce the imaging system weight (Cui Hongxia, 2008).

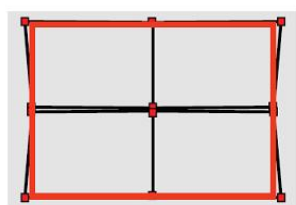

a DMC

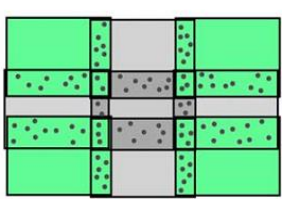

b UltraCamD

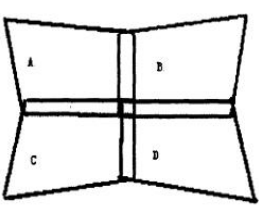

c SWDC
Figure 2. Mechanical structures of DMC,UCD,SWDC cameras

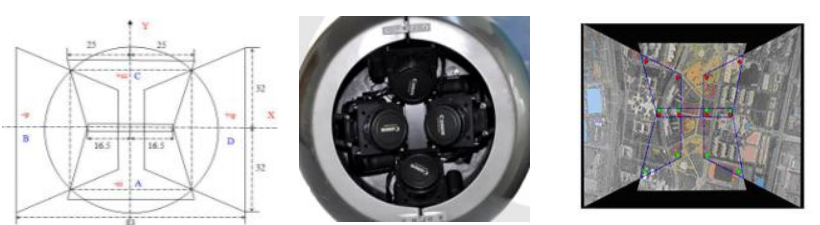

Figure 3 . The structure of the designed four-combined wideangle camera(Unit: $\mathrm{mm}$, view angle: $100^{\circ} \times 130^{\circ}$ )

\subsection{Precise orientation}

3.2.1 Self-calibration of four-combined camera: In order to meet the UAV load requirements, four-combined camera adopt the special light and small mechanical structure, so the image displacement caused by mechanical deformation is relatively large, which affects the image splicing quality. Self-calibration of four-combined camera can find the camera minor changes to correct, obtain accurate orientation relationship between cameras and reduce image splicing error (Liu Zhaoqing, 2008).

Before the self-calibration of four-combined camera is done, every single camera must be calibrated to obtain its precise inner orientation elements. The outdoor calibration filed in the Chinese Academy of Surveying and mapping is used for single camera calibration, which is shown in Fig.4. Corresponding image points got by feature matching in the image overlap area are used to calibrate the images relative exterior orientation elements, which can eliminate the independent error in every single camera and ensure the spliced images without obvious misplacement or splicing seam. Selfcalibration process of four-combined camera is shown as in Fig. 5 .

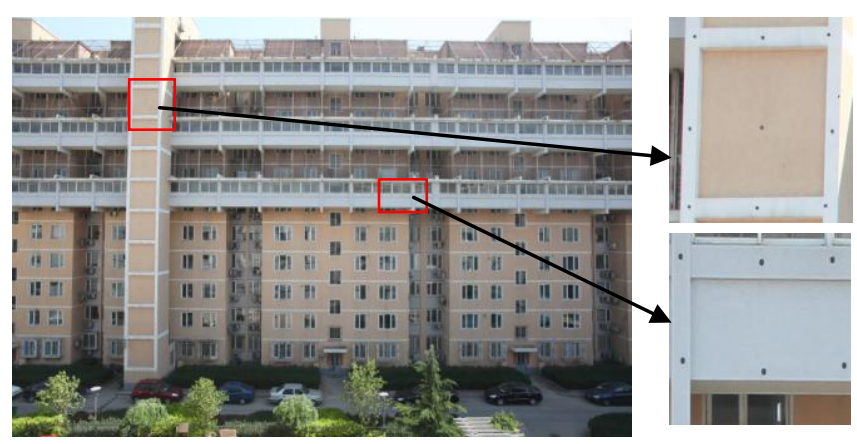

Figure 4 . The outdoor calibration filed 


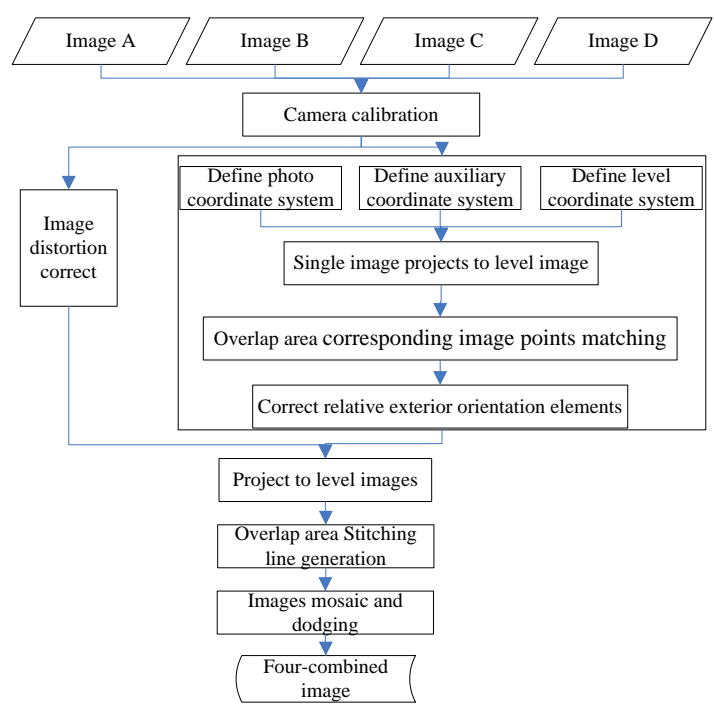

Figure 5. Self-calibration process of four-combined camera

According to the self-calibration process of four-combined camera, the calibration result is calibrated in Table 1 and the splicing image is shown as in Fig.6b. It shows that the errors of every camera's exterior orientation elements which is calibrated by space resection are independent and close to 2 pixels. After self-calibration, the system error can be eliminated and the mean error of the image splicing can be limited to 0.2 pixels, so the precise relative relationship between cameras is established.

\begin{tabular}{c|c|c|c|c|c}
\hline $\begin{array}{c}\text { Overlap } \\
\text { area }\end{array}$ & $\begin{array}{c}\text { Number of } \\
\text { matching } \\
\text { points }\end{array}$ & $\begin{array}{c}\text { residual er } \\
\text { ror before } \\
\text { self- } \\
\text { calibration } \\
\text { (Ex) }\end{array}$ & $\begin{array}{c}\text { Residual } \\
\text { error after } \\
\text { self- } \\
\text { calibration } \\
\text { (Ex) }\end{array}$ & $\begin{array}{c}\text { Mean } \\
\text { error } \\
\text { before } \\
\text { self- } \\
\text { calibratio } \\
\mathrm{n}\end{array}$ & $\begin{array}{c}\text { Mean } \\
\text { error } \\
\text { after } \\
\text { self- } \\
\text { calibratio } \\
\mathrm{n}\end{array}$ \\
\hline $\mathrm{AB}$ & 3353 & 0.1838 & -0.1707 & 2.1938 & 0.1775 \\
\hline $\mathrm{AC}$ & 116 & 0.4715 & -0.0488 & 2.3258 & 0.1775 \\
\hline $\mathrm{AD}$ & 2691 & -0.2921 & -0.0761 & 1.9314 & 0.1775 \\
\hline $\mathrm{BC}$ & 3413 & -0.3314 & -0.0545 & 2.0195 & 0.1775 \\
\hline $\mathrm{CD}$ & 2280 & -0.2821 & -0.0188 & 2.0471 & 0.1775 \\
\hline
\end{tabular}

Table 1. Result of self-calibration

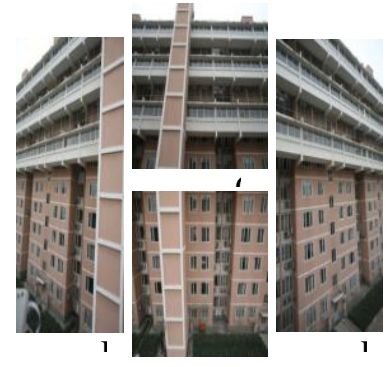

Figure 6a. Images from fourcalibrated
3.2.2 Aerotriangulation with four-combined images: Aerotriangulation process with four-combined images is image feature point extraction relative orientation, absolute orientation and block adjustment to obtain the high precision attitude parameter and orientation elements, so according to the selfcalibration result, the exterior orientation elements of every camera's images can be calculated.

The UAV platform requirement for light, small and flight low can't avoid the phenomenon that the images have big pitch, roll and yaw(Pitch and roll are over than $5^{\circ}$. Yaw is over than $15^{\circ}$ ). Images with big pitch and roll lead to the same feature in different images with large geometric deformation and their gray values obvious difference, which increases the matching difficulties. If yaw is over than $15^{\circ}$, the image matching is often not successful.

According to the characters of four-combined images, we combine with the existing image matching methods and study a new method of stratified matching based on image pyramid to solve the image matching problem. Firstly, images are built to two levels of pyramids. On the top pyramid images, SIFT (Scale Invariant Feature Transform) image matching is done to obtain matching points. Then, the homography matrix which is used for compensate the image geometric distortion is calculated with the matching points by random sample consensus (RANSAC) algorithm. On the bottom pyramid images, Harris feature extraction is used to obtain many feature points and the SIFT feature points on the top pyramid images are passed down. Then, bidirectional concordance correlation coefficient algorithm is used for feature points dense matching. After that, the homography matrix is updated, which is used for delete false matching points by setting threshold. Finally, corresponding image points are up to sub-pixel accuracy by least squares matching (LSM). The technological process is shown as in Fig.7.

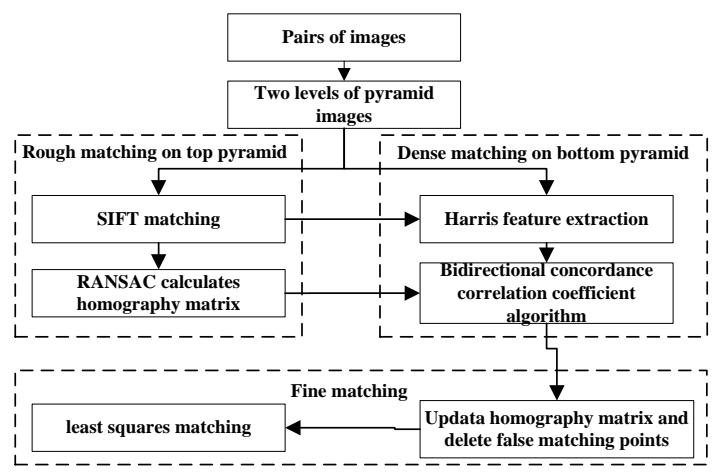

Figure 7. Image matching process

\subsection{Building measurement}

Building measurement mainly includes building roof contour acquisition and building side details measurement.

With aberration rectification and horizontal projection, fourcombined images can be used as source data of building roof contour extraction, the buildings in which are most close to the vertical photography. Because the resolution of Low altitude aerial images is enhanced, the feature details on images are more complex. So, errors that building roof contours are 
automatic extracted increase the difficulty of follow-up manual amendment. Nowadays, Semi-automatic roof contour extraction is the most effective method. In the paper, firstly, we use artificial building target identification and separate point positioning for rough localization, which can greatly reduce the workload of manual clicks. Then, after feature matching, the matching points are used for automatic buildings measurement by least squares indirect adjustment based on the geometric constraints of the object aspect (Coplanar, vertical, parallel, equidistant, same highs and collinearity etc.) (Roth.G, 2004). Fig. 8 shows several typical building roofs by semi-automatic measurement in the stereo image, in which the red circle is the initial positions by the clicks of the mouse.
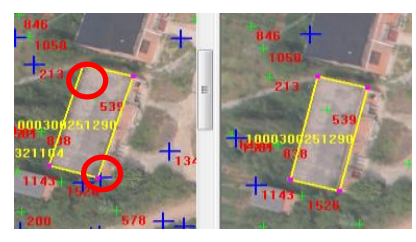

Figure 8a. The quadrangle and right-angle roof building measurement

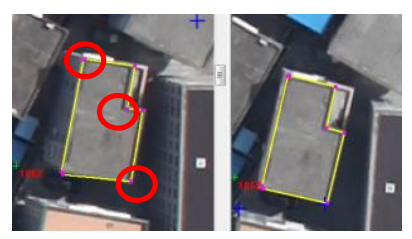

c. The polygon and right-angle roof building measurement

In building 3D model construction, building edge corner measurement is the basis of geometric model construction. The biggest advantage of UAV carrying four-combined camera is that vertical and inclined images of one building can be got at the same time by route planning, which avoids photography shielding and sheltered corner problem with binocular vision and improves the accuracy of measurement adjustment with redundant condition of multiple visual observation (Gui Dezhu, 2010). These provide possibility for building precise details measurement. Three-dimensional coordinates of corner points imaging in two or more images can be calculated by multiple tablets combined bundle adjustment method. Then the corner point in the image which is selected with the largest area or the minimum shading area of the wall is used for $3 \mathrm{D}$ model construction, and other corner points is used for check.

\subsection{Building texture collection}

Building texture collection includes roof texture collection and wall texture collection.

Based on the known internal and external orientation elements, four-combined images, in which the buildings are most close to the vertical photography and the roofs are less occluded, can be used for roof texture collection through calculating the building corner point with the images coordination according to the point with the object coordination by point counter cast method. Point counter cast method is shown as bellow.

$$
\begin{aligned}
& x-x_{0}=-f \frac{a_{1}\left(X-X_{s}\right)+b_{1}\left(Y-Y_{s}\right)+c_{1}\left(Z-Z_{s}\right)}{a_{3}\left(X-X_{s}\right)+b_{3}\left(Y-Y_{s}\right)+c_{3}\left(Z-Z_{s}\right)} \\
& y-y_{0}=-f \frac{a_{2}\left(X-X_{s}\right)+b_{2}\left(Y-Y_{s}\right)+c_{2}\left(Z-Z_{s}\right)}{a_{3}\left(X-X_{s}\right)+b_{3}\left(Y-Y_{s}\right)+c_{3}\left(Z-Z_{s}\right)}
\end{aligned}
$$

Where: $x, y=$ the coordinates of the building corner point with photo coordinate system;

$x_{0}, y_{0}, f=$ internal orientation elements;

$X_{s}, Y_{s}, Z_{s}=$ the coordination of the photo site with object space coordinate system;

$X, Y, Z$ =the coordination of the building corner point with object space coordinate system;

$a_{i}, \quad b_{i}, \quad c_{i}(i=1,2,3)=$ the nine direction cosines combined by three external orientation elements.

Wall textures are often collected by photographing the wall on the ground and then using artificial texture mapping, which is low effective. With the distance constraints of camera to building, occlusion is serious and it is difficult to obtain the wall "panorama" (Zhang Zuxun, 2003). Thank to UAV flying low, many building side textures can be obtained with high resolution, which provide new data source for building texture collection. The process of building texture collection is shown as bellow.

1) Many wall textures from multi-view images are obtained by point counter cast method;

2) Some better images are selected by view angle method. View angle method is that every side $T_{K}$ in the building geometry model is processed as bellow:

a calculating unit normal vector $n /|n|$ of this side;

b for every image $i \quad(0 \leq i \leq M)$, calculating view direction $N_{i}$ and the space angle $\theta_{i}$ between $N_{i}$ and $n /|n|$;

c comparing the calculated angle $\theta_{i}$ of $\mathrm{M}$ images and making the index $i$ of the image with minimal angle to this side ${ }^{T_{K}}$

3) Depth buffer algorithm (Famhi A, 1998) is used to detect whether the building shielded or not. If it is, the image with second minimal angle is elected as the best wall texture.

\section{EXPERIMENTS AND ANALYSIS}

The first 3D building construction experiment is in Shandong University of Science and Technology, which uses unmanned airship FKC-1 carrying four-combined camera for aerial photography. Utilizing the high precision aerial experimental control filed in the university (total 20 control points), this aerial photography meets the aerial photogrammetry accuracy with scale mapping of 1:1000.

Using the method mentioned in this paper, the buildings such as teaching building, dormitory and canteen are processed with $3 \mathrm{D}$ measurement, texture acquisition and 3D model construction. 3D building geometric model is shown in Fig.9 and 3D effect graph of the teaching building is shown in Fig.10. 


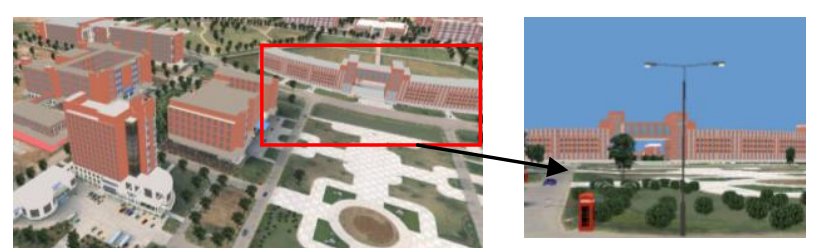

Figure 9. 3D building geometric Figure 10. 3D effect model graph of the teaching building

The second 3D building construction experiment is in Henan University of Urban Construction, which uses fixed wing UAV carrying four-combined camera for aerial photography. Utilizing intensive control points (total 36 control points) in the university, this aerial photography meets the aerial photogrammetry accuracy with scale mapping of 1:500. 3D building geometric model is shown in Fig.11 and 3D effect graph of the library building is shown in Fig. 12.

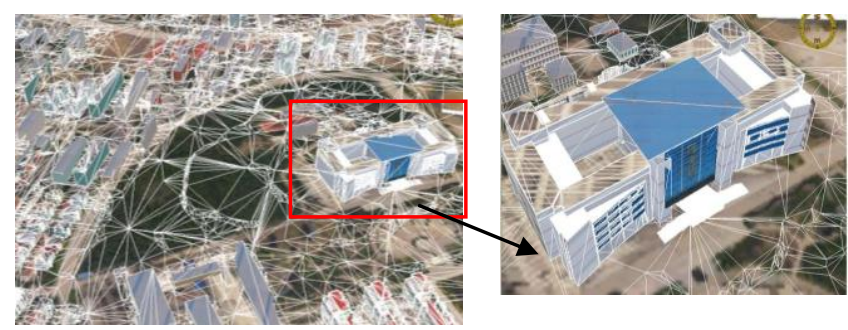

Figure 11.3D building geometric model

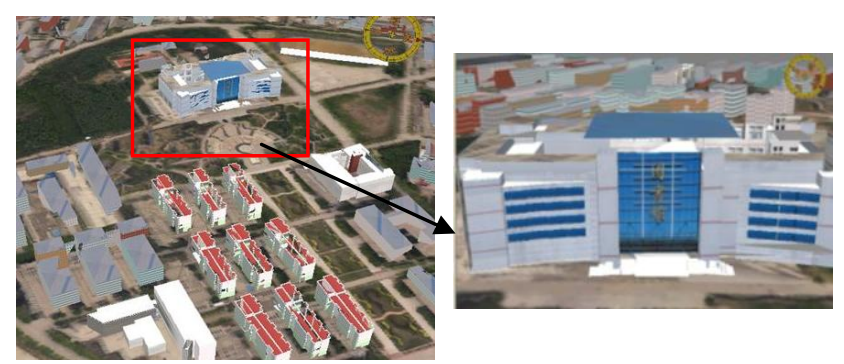

Figure 12.3D effect graph of the library building

\section{CONCLUSION}

In this paper, a method of fast 3D building modeling using the images from UAV carrying four-combined camera is studied. Two experiments that are aerial photography with large scale mapping of 1:1000 and 3D building construction in Shandong University of Science and Technology and aerial photography with large scale mapping of 1:500 and 3D building construction in Henan University of Urban Construction are done. It is demonstrated that UAV carrying four-combined camera for low altitude aerial photogrammetry can meet the accuracy requirement of the mapping scale of 1:1000 and 1:500 standard, and effectively solve the city complex building 3D modeling texture acquisition, which has better application prospect.

\section{REFERENCES}

Besnerais, G, Sanfourche, M.2008. Dense height map estimation from oblique aerial image sequences. Computer Vision and Image Understanding, 109(2),pp204-225.

Cui Hongxia, Lin Zongjian, Yang Hong. 2008. Multiview photogrammetry using low altitude digital images from unmanned airship. Opto-Electronic Engineering, 35(7),pp7375.

Famhi A, Josef J, Christine R.1998. The Generation of True Orthophotos Using a 3D Building Model in Conjunction With a Conventional DTM.ISPRS Commission IV "GIS Between Visions and Applications".

Gui Dezhu. 2010. Study on construction of 3D building based on wide-angle and combine camera images from UAV. Ph.D. Thesis, Chinese University of Mining and Technology.

Li Chengming, Wang Jizhou, Ma Zhaoting. 2008. Digital city geographic space frame principles and methods. Beijing, Science Press.

Lin Zongjian.2011. UAV borne low altitude photogramm etry system. Science of Surveying and Mapping, 36(1),pp.5-9.

Liu Zhaoqin. 2008. Study on wide-angle and light small combined digital camera system for UAV. Ph.D. Thesis, Shandong University of Science and Technology.

National Remote Sensing Center, 2009. Advance in Geo-spatial Information Science and Technology. Beijing, Publishing House of Electronics Industry.

Peng Xiaodong, Lin Zongjian.2009. Unmanned airship low altitude system for aerial photogrammetry. Science of Surveying and Mapping, 34(4),pp33-35.

Roth,G. 2004. Automatic correspondences for photogrammetric model building. International Archives of Photogrammetry Remote Sensing Spatial Information Science, 35(B5),pp713718 .

Wu Jun. 2003. Research on Rapidly Reconstructing Texture for Facades in 3D City Modeling. Ph.D. Thesis, Wuhan University.

Zhang Zuxun, Zhang Jianqing.2003. Solutions and core techniques of city modeling. World Sci-tech $R \& D$, 25(3),pp23-29. 